\title{
A Multicast Extension for Enhanced Mobile IP by Home Agent Handover
}

\author{
Chun-Chuan Yang ${ }^{1}$, Jeng-Yueng Chen ${ }^{1,2}$, and Li-Sheng $\mathrm{Yu}^{1}$ \\ ${ }^{1}$ Department of Computer Science and Information Engineering, \\ National Chi Nan University, Puli, 545 Nantou, Taiwan \\ ${ }^{2}$ Department of Information Management, \\ Hsiuping Institute of Technology, Dali, 412 Taichung, Taiwan \\ \{ccyang, s2321902, s2321535\}@ncnu.edu.tw
}

\begin{abstract}
In order to improve the routing efficiency and reduce handoff latency, we have proposed an enhancement of Mobile IP (MIP) called MIP with Home Agent Handover (HH-MIP) to enjoy most of the advantages of Route Optimization MIP (ROMIP) but with only a small increase of signaling overhead. In HH-MIP, the concept of Temporary Home Agent (THA) was proposed and the mobile host $(\mathrm{MH})$ registers the new CoA with its THA rather than its original HA. In this paper, we propose a multicast extension for $\mathrm{HH}$ MIP (HH-MIP/ME). HH-MIP/ME reduces routing inefficiency in bi-directional tunneling without generating large signaling overhead. Simulation results demonstrate that the proposed scheme enjoys small handoff latency as well as routing efficiency and the number of control packets generated in proposed scheme is significantly less than that in other approaches.
\end{abstract}

Keywords: Mobility, Multicast, Mobile IP, Route Optimization.

\section{Introduction}

Mobility management in the IP layer [1] is an essential component in wireless mobile networking. Mobile IP (MIP) [2]-[3] was proposed to support global Internet mobility through the introduction of location directories and address translation agents. In the MIP, a mobile host (MH) uses two IP addresses: a fixed home address and a care-ofaddress (CoA) that changes at each new point of attachment. A router called Home Agent (HA) on an MH's home network is responsible for maintaining the mapping (binding) of the home address to the CoA. When an MH moves to a foreign network, the $\mathrm{MH}$ obtains a CoA from the Foreign Agent (FA) and registers the CoA with its HA. In this way, whenever an $\mathrm{MH}$ is not attached to its home network, the HA gets all packets destined for the $\mathrm{MH}$ and arranges to deliver to the MH's current point of attachment by tunneling the packets to the MH's CoA. Some inefficiencies were identified in MIP: (1) Triangular routing from the sender (called correspondent node, $\mathrm{CN}$ ) to the HA then to the mobile host leads to unnecessarily large end-to-end packet delay, (2) The HA is inevitably overloaded due to tunneling operations, and (3) When an $\mathrm{MH}$ is far away from its home network, the long signaling path for CoA registration leads to a long handoff latency resulting in a high packet loss. [4]-[5] 
To remedy the problem of triangular routing and reduce the packet loss during handoff, Route Optimization MIP (ROMIP) [6]-[7] was proposed. The ROMIP allows every $\mathrm{CN}$ to cache and use binding copies. The original binding for an $\mathrm{MH}$ is kept in its HA, but the ROMIP supports that a binding copy can be propagated to the requiring nodes. Local bindings in a $\mathrm{CN}$ enable most packets in a traffic session to be delivered by direct routing. Moreover, an $\mathrm{MH}$ also informs its previous FA about the new CoA, so that the packets tunneled to the old location (due to an out-of-date binding copy) can be forwarded to the current location. This forwarding mechanism in ROMIP reduces the handoff latency and thus reduces the packet loss during handoff. However, the improvement of ROMIP over MIP in terms of routing efficiency and smaller handoff latency is at the cost of significantly larger signaling overhead.

An interesting point of view about the reason of the disadvantages of MIP in routing and handoff latency is because the $\mathrm{MH}$ has the potential to move away from its home network and the HA. If somehow we can dynamically make the HA closer to the current location of the $\mathrm{MH}$, both routing and handoff efficiency can be achieved. Since the MH's home address is permanent, MH's HA should not move. Therefore, the idea of Temporary HA (THA) emerged and the extension of MIP adopting the THA called HA Handover MIP (HH-MIP) was proposed in [8]. The HH-MIP enjoys small handoff latency as well as routing efficiency and the number of control packets generated in the HH-MIP is significantly less than that in the ROMIP.

With the development of communication and multimedia technology, applications that use multicast as transmission method become more and more popular. However, the MIP is designated for unicast delivery to MHs. To perform multicast functionality, additional mechanisms must be added to the protocol to efficiently support multicast delivery within or on top of the MIP. The current version of the MIP proposes two approaches, called Remote Subscription (RS) and Bi-directional Tunneling (BT) [9], to support mobile multicast.

In the RS, the $\mathrm{MH}$ has to re-subscribe to its desired multicast groups while the $\mathrm{MH}$ moves to a new foreign network. This mechanism works well when the MH spends a relatively long time at each foreign network, compared with the join and graft latencies. The advantage of RS is it delivers multicast packets to related $\mathrm{MHs}$ in shortest path routes. However, RS introduces excessive control packets and packet loss because it needs to reconstruct multicast tree every time the $\mathrm{MH}$ moves to new foreign network.

With the BT, the $\mathrm{MH}$ sends and receives all multicast datagram from its HA. Multicast packets will be sent to the MH's HA and tunneled to current position of the MH using MIP unicast tunneling. This approach handles source mobility as well as recipient mobility, and in fact hides host mobility from all other members of the group. The main disadvantages of the protocol are the routing path for multicast delivery can be far from optimal (in the worst case, the source and the recipient can be on the same network, while all multicast messages between two hosts must traverse to the home agent before tunnel to the designated network) and the approach offers limited scalability. Some of the enhancement protocols based on bi-directional tunneling are Mobile Multicast (MoM) [10], Mobile Multicast Gateway (MMG) [11] and Range-base Mobile Multicast Protocol (RBMoM) [12]. 
In this paper, we introduce multicast extension based on our proposed mobility management protocol (HH-MIP) called HH-MIP Multicast Extension (HH-MIP/ME). Since the proposed multicast protocol is based on the HH-MIP, it will inherit the advantages of the HH-MIP. The proposed approach is a hybrid of RS and BT. We also use the idea developed from the MoM called Designated Multicast Service Provider (DMSP) [10]. The DMSP is used to solve the data duplication and tunnel convergence problem. The HH-MIP/ME reduces routing inefficiency in bi-directional tunneling without generating large signaling overhead. As will be shown in the simulation study, the HH-MIP/ME also enjoys small handoff latency as well as routing efficiency and the number of control packets generated in the HH-MIP is significantly less than that in the RS and BT-based MoM.

The rest of this paper is organized as follows. Section 2 reviews the HH-MIP approach. The proposed multicast extension of the HH-MIP is presented in section 3 . Simulation studies for performance evaluation and comparison are presented in section 4 . Finally, section 5 concludes this paper.

\section{HH-MIP Approach}

As mentioned in section 1, the HH-MIP introduces the concept of Temporary HA (THA) and as in the ROMIP each $\mathrm{CN}$ is required to maintain two addresses for an $\mathrm{MH}$ : the home address of the $\mathrm{MH}$ and the THA address of the MH. The HA of an MH maintains the binding of the THA address for the MH. Handover of the THA requires the $\mathrm{MH}$ to update the binding cache in its HA. The handoff of an MH to a new FA only triggers registration of the new CoA to the THA (instead of the HA) when the THA of the MH remains unchanged. Since the THA of an $\mathrm{MH}$ is selected to be close to the current location of the $\mathrm{MH}$, the HH-MIP reduces the handoff latency and shortens the signaling path of registration as well.

Data delivery in the HH-MIP is similar to that in the ROMIP as explained in the following. Initially the $\mathrm{CN}$ sends packets to the home address of the destined $\mathrm{MH}$, the HA intercepts and sends the packets to the THA by tunneling, and the THA tunnels the packets to the current location (FA) of the MH. Meanwhile, a binding copy of the MH's THA is sent by the HA to $\mathrm{CN}$ so that later packets can be directly delivered to the THA, and the THA tunnels the packets to the current location (FA) of the MH. Therefore, regular data delivery in the HH-MIP requires the packets sent by the $\mathrm{CN}$ to be tunneled twice before they reach the destined $\mathrm{MH}$.

Four messages are used for binding update of THA as in ROMIP: (1) Binding Warning Message $\left(M_{W}\right)$, (2) Binding Request Message $\left(M_{R}\right)$, (3) Binding Update Message $\left(M_{U}\right)$, and (4) Acknowledgement Message $\left(M_{A}\right)$. The HA just after having tunnels the first packet sends an $M_{W}$ back to the $\mathrm{CN}$ informing that the $\mathrm{MH}$ is not in the home network. In response to the received $M_{W}$, the $\mathrm{CN}$ sends an $M_{R}$ to the HA asking for binding update. The HA replies with an $M_{U}$ containing the requested CoA (i.e. THA's address). Finally, the $\mathrm{CN}$ sends an $M_{A}$ to the HA acknowledging the successful binding update. Fig. 1 (a) illustrates the process of data delivery in the HH-MIP. 


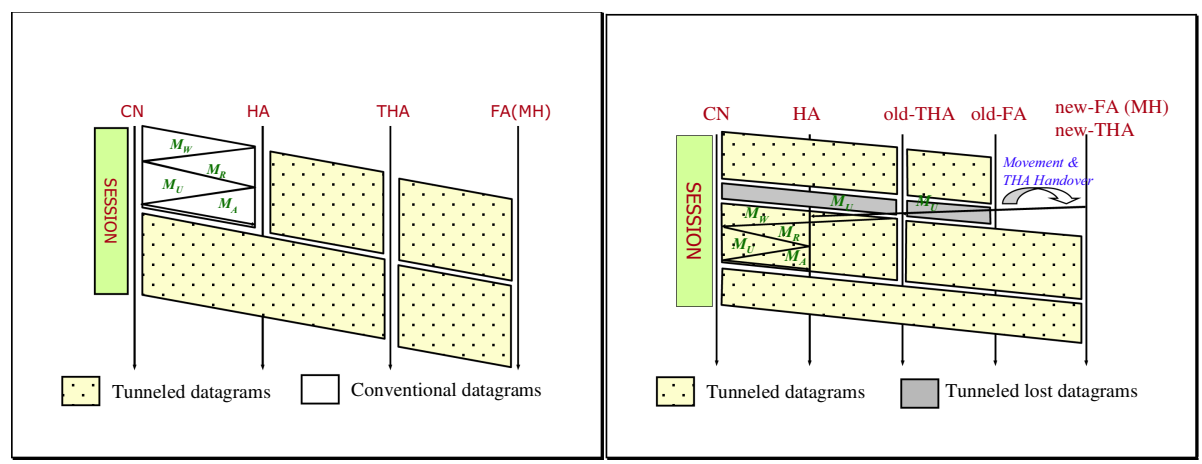

(a) without THA handover

(b) with THA handover

Fig. 1. Flow diagram for data delivery in HH-MIP

Initially, an MH will select its HA as the THA. The HH-MIP adopts an aggressive approach in selecting the THA for an $\mathrm{MH}$ : whenever an $\mathrm{MH}$ is moving away from the HA or the previous THA, the MH triggers the handover of THA. If the distance (hop count) from FA2 (MH's current location) to THA is longer than the distance from FA1 to THA implying that the MH is moving away from THA, FA2 is selected as the new THA, and the MH notifies its HA of the new THA. On the other hand, if HA is closer to FA2 than THA implying that the MH is moving back to HA, the HA should be selected as the new THA.

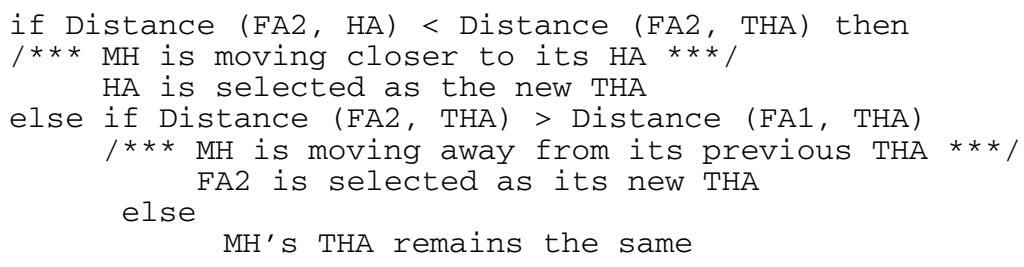

Once a new FA is selected as the new THA by an MH, the MH sends the Binding Update Message $\left(M_{U}\right)$ to its HA as well as the previous THA. Before the CN gets the address of new THA (according to the $M_{U}$ sent by the HA), packets are still tunneled to the previous THA (packets loss in this period), and the previous THA tunnels (forwards) the packets to the current FA (i.e. the new THA) which is similar to the forwarding mechanism in ROMIP. When the binding update of the new THA is complete in the $\mathrm{CN}$, packets are sent directly to new THA. Flow diagram for the handover of THA is illustrated in Fig. 1 (b).

The HH-MIP adds new functional entity called Temporary Home Agent (THA) besides functional entities introduced in Mobile IP. Each FA or HA must be equipped with the functions of THA. The functions of the THA include: (1) maintaining a Temporary Children List (TCL) and dealing with the registration of the new CoA for every MH in the TCL, and (2) a previous THA for an MH is responsible for forwarding packets to the new THA after the MH performs THA handover. 
HH-MIP also includes messages type that is similar to ROMIP. These messages include:

1. Binding Warning Message $\left(M_{W}\right)$ - is sent to inform the target nodes about changing THA.

2. Binding Update Message $\left(M_{U}\right)$ - is used to inform $\mathrm{CN}$, old THA, or HA about the new THA address. THA can send $M_{U}$ messages without waiting for request message.

3. Binding Request Message $\left(M_{R}\right)$ - is sent by $\mathrm{CN}$ when it determines that its binding is stale and it wants to request connection to the THA.

4. Binding Acknowledge Message $\left(M_{A}\right)$ - is used to acknowledge the reception of binding update message $\left(M_{U}\right)$. Not every binding update need to be acknowledged.

\section{Proposed HH-MIP/ME Approach}

\subsection{Basic Idea and Data Delivery}

As we build our multicast extension on the top of HH-MIP, MH relies on THA to forward multicast traffic to $\mathrm{MH}$ through the tunneling via the FA. In this approach, THA will join multicast group on behalf of $\mathrm{MH}$. As $\mathrm{MH}$ moves to other FA (without THA handover), multicast packets will be sent to THA and THA will tunnel the packets to MH's current position (FA). As illustrated in Fig. 2, THA will join multicast group on behalf of MH. Multicast Sender sends multicast packets to THAs by using multicast address. After receiving multicast packets, THAs will tunnel the multicast packets to MH's current FAs. FAs will complete multicast packets delivery to MHs by using link-level multicasting.

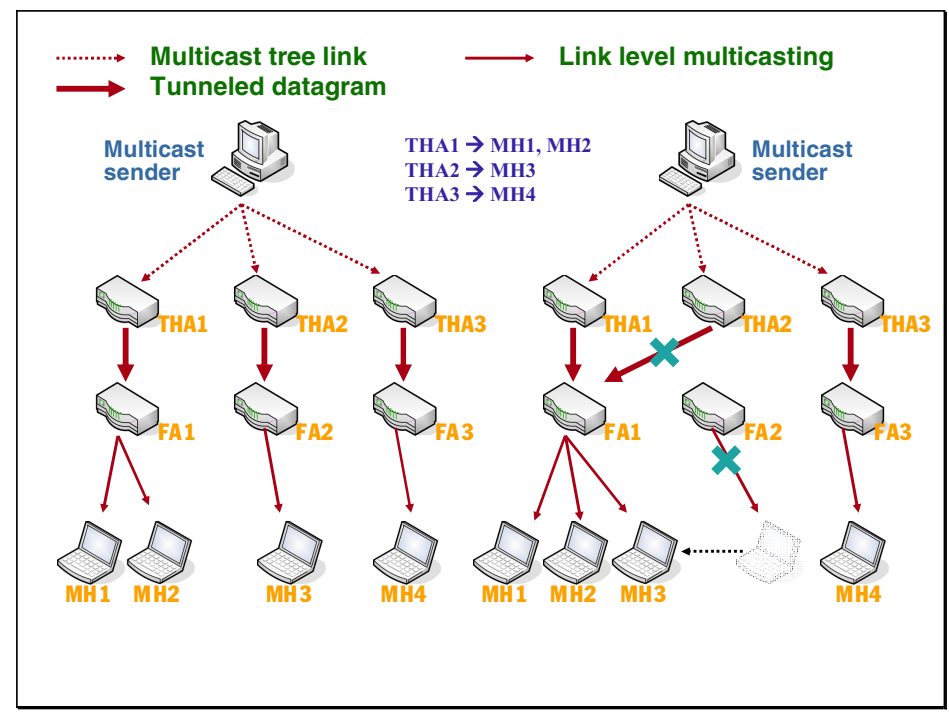

Fig. 2. HH-MIP/ME Data delivery 
To avoid the duplication of multicast packets on the foreign network in the event that the THA has multiple MHs present there, just one copy of multicast datagram is sent to the foreign network and link-level multicasting is used by the FA to complete the delivery.

To solve the tunnel convergence problem, the FA will select one of the THAs as the DMSP, for a given multicast group. THAs that are not the DMSP for a given multicast group can suppress delivery down the tunnel using negative caching, as described in PIM [13]. DMSP handover must be performed in case the MH that owns current active DMSP moves from current network to another foreign network. For DMSP selection, we use the THA that has been in the THA list for the longest time.

In THA handover scenario, MH will have to rejoin multicast group (if new FA has not joined the multicast group yet) by sending IGMP [14]-[15] join message to reconstruct multicast tree. In case current network (new FA) is served by DMSP, THA will suppress the multicast delivery of serving DMSP by sending negative caching. After completing the multicast tree, multicast sender will send multicast datagram directly to THA. Multicast tree in old THA will be deleted if no other MHs required. In case that the MH's old THA is the DMSP for a group at the (previous) foreign network, a DMSP handoff is required to select new DMSP and to forward datagram to the remaining multicast group members (if any) at the (previous) foreign network. Until the DMSP handoff is completed, multicast delivery for group members at the foreign network will be disrupted. Fig. 3 illustrates the join message and data delivery during THA handover.

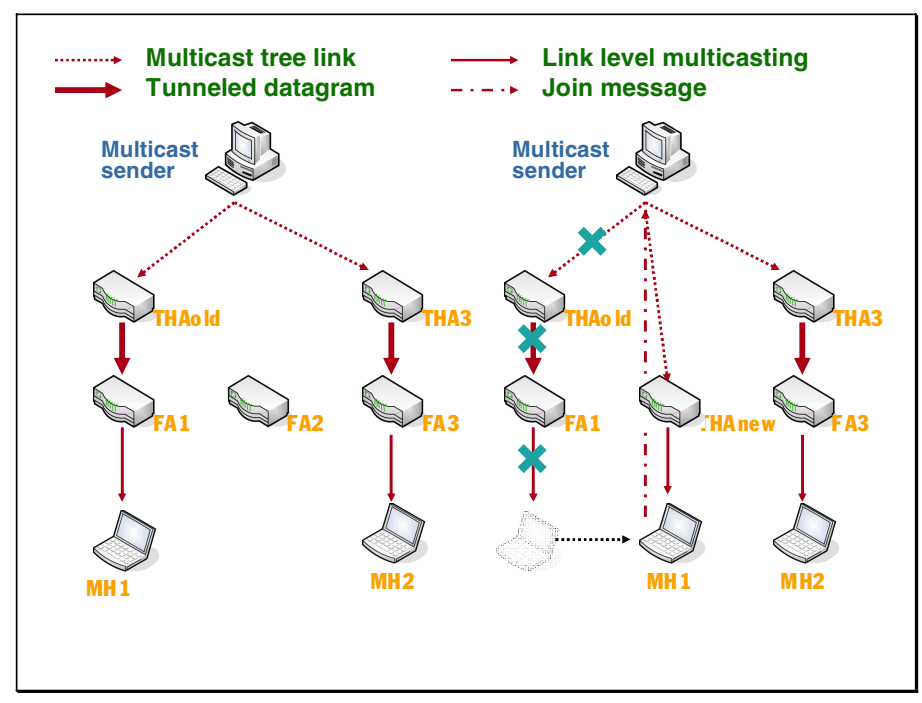

Fig. 3. Join message and data delivery during THA handover

\subsection{Protocol Data Structure}

In order to support HH-MIP/ME, data structures included in HH-MIP protocol must also be supported. These data structures include: (1) each HA must maintain an away 
list. Away list is used to keep track of which of its own MHs are away and their current active THA, (2) each FA maintains a visitor list. Visitor list is used to keep track of which mobile hosts are currently at its LAN and their current active THAs, and (3) each THA must maintain Temporary Child List (TCL) to keep track of temporary MHs that THA has responsible, from where these mobile hosts come from and where these mobile hosts are. In HH-MIP, THA can be an FA or HA.

The HH-MIP/ME protocol also requires group membership information for the away and visiting MHs. Each THA Group Information keeps track of three things for each multicast group that it knows about: a list of away MHs that are members of the group, a list of the FAs at which the away group members reside, and a list of the FAs for which the THA has DMSP responsibilities. Similarly, each FA keeps track of three things on a per group basis: a list of visiting mobile hosts that are members of the multicast group, a list of the THAs to which these visiting group members belong, and a list of THAs that are currently serving as DMSPs for this group.

\section{Performance Evaluation}

\subsection{Simulation Environment}

The network topology in our simulation is $8 \times 8$ mesh network. Each node in the mesh represents an FA. The locations of the HA and multicast sender are randomly selected from the mesh. Initial locations for the MHs are also randomly selected from the mesh. In order to model the mobility of the MHs, time is slotted and the parameter called Movement Probability (MoveProb) [16] is used in the simulation. MoveProb represents the probability that an $\mathrm{MH}$ leaves its current network in the next slot time. Thus, we could model high mobility of MHs by assigning a large value of MoveProb. When an $\mathrm{MH}$ decides to leave the current network in the next slot time, its next foreign network is randomly selected from the neighboring networks. For simplicity of our simulation, there is only one multicast group in which only one multicast source is assumed. The number of MHs in multicast group varies from 10 to 50 . We also simulate the protocols performance in different mobility pattern. Total run time in the simulation for each approach is 500 slot times.

\subsection{Performance Criteria}

In the performance evaluation, we compare our approach with MoM (bi-directional tunneling enhancement using DMSP) and remote subscription (RS). Both protocols have their own advantage and disadvantage. MoM is based on MIP which multicast packets are received by $\mathrm{HA}$ on behalf of $\mathrm{MH}$. This approach handles source mobility as well as recipient mobility and in fact hides host mobility from all other members of the group. The disadvantages of this approach are the routing path for multicast delivery can be far from optimal and scalability problem. With remote subscription, the $\mathrm{MH}$ has to re-subscribe multicast group when it moves to other domain network. The main advantages of remote subscription are it is a simple protocol and it has the 
optimal path for multicast packets delivery. The drawbacks for remote subscription are it introduces large signaling overhead and large packet loss as MH moves to different domain network.

Some criteria are used to compare the performance of our approach with other approaches:

1. Average of the summation of end-to-end path length in a group. In average of the summation of end-to-end path length in a group (in hop counts), we will measure the average number of hop counts the multicast packets travel from multicast source to each MH's current position. End-toend path length is used to show the routing efficiency of the approaches. The longer the distance the packets travel from sender to destination means the larger the delay between sender and receiver. Approach with large end-to-end delay is not suitable for real time interactive applications.

2. Tree maintenance overhead. In tree maintenance overhead (in average number of control packets), we will measure the average number of join and leave messages have been sent during the simulation period. For $\mathrm{HH}-$ MIP/ME, join message is sent when MH arrives in new foreign network (FA has not joined multicast group membership yet) or when $\mathrm{MH}$ triggers the THA handover. Leave message is sent when MH leaves previous network and no other MHs use the multicast tree. Comparison will be made between HH-MIP/ME and remote subscription.

3. Average number of DMSP handoff. In average number of DMSP handoff (in number of DMSP handoff), we will measure the average number of DMSP handoff per MH handoff during the simulation period. Increase in DMSP handoff will introduce packet loss because packet loss will occur before DMSP handoff is completed. Comparison will be made between HHMIP/ME and MoM.

4. Average DMSP handoff latency. In average DMSP handoff latency (in hop counts), we will measure the average number of hop counts the DMSP update message travels from foreign network to new selected DMSP. Longer path will induce larger packet loss caused by longer DMSP handoff time. Comparison will be made between HH-MIP/ME and MoM.

\subsection{Simulation Results}

The first simulation result is to evaluate the routing efficiency of different approaches. In average end-to-end path length, we compare the end-to-end routing path of multicast packets between each protocol. Fig. 4 shows the end-to-end path length of different protocols at different group member size. Remote subscription has the best performance compare to the other protocols because each multicast delivery is sent directly to its current foreign network. The HH-MIP/ME has a better performance than MoM because multicast packets are sent to the THA which resides near mobile host current position. As for MoM, it has the worst performance because of the triangular routing problem. 


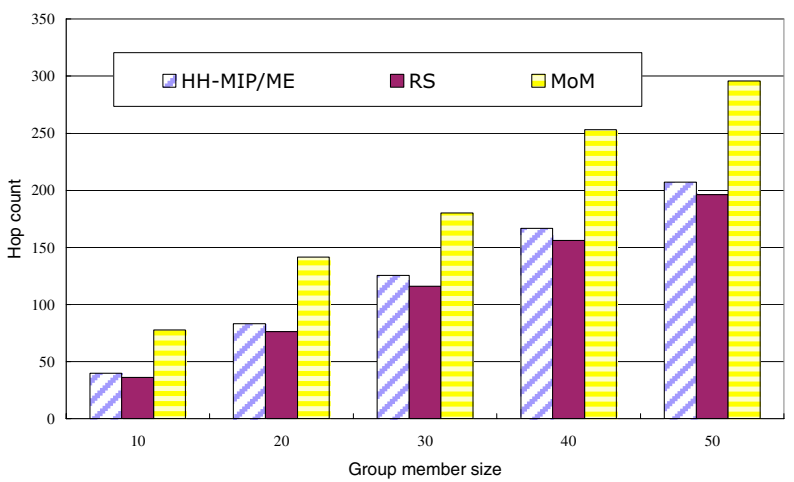

Fig. 4. Summation of end-to-end path length in a group

Fig. 5 compares the tree maintenance overhead between HH-MIP/ME and Remote Subscription. The overhead of concern is join and leave messages have been sent during multicast tree reconstruction. Remote Subscription introduces larger overhead because $\mathrm{MH}$ needs to send join message each time it moves to different network and sends leave message to quit from the tree. Obviously, the tree maintenance cost will increase when the mobility is getting higher. For the HH-MIP/ME, it reduces the overhead by reducing unnecessary tree reconstruction. The HH-MIP/ME will need to reconstruct multicast tree in case of THA handover. The HH-MIP/ME also performs well in case of high mobility.

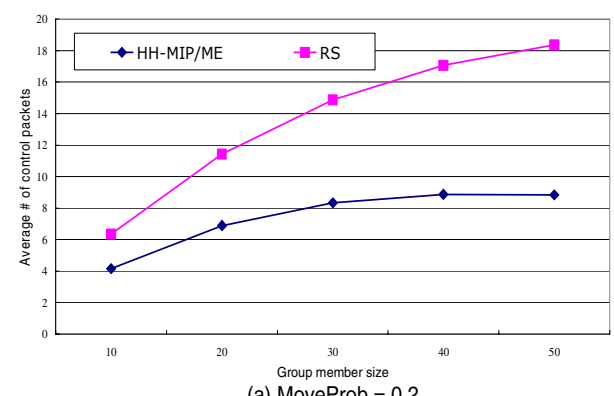

(a) MoveProb $=0.2$

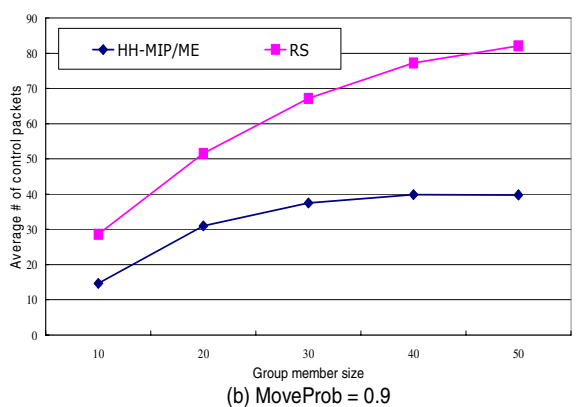

(b) MoveProb $=0.9$

Fig. 5. Tree maintenance overhead at different MoveProb

Fig. 6 shows the average number of DMSP handoff during the total simulation period in HH-MIP/ME and MoM. In high mobility, the DMSP will handoff more frequently since MHs' handoff take place easily. MoM has fewer DMSP handover because HA that acts as DMSP is static. The DMSP will handoff only if the DMSP owner leaves its current network. The HH-MIP/ME has larger number of DMSP 
handoff because THA handover has the chance to initiate the DMSP handoff. Number of DMSP handoff in both protocols will increase in case of high mobility.

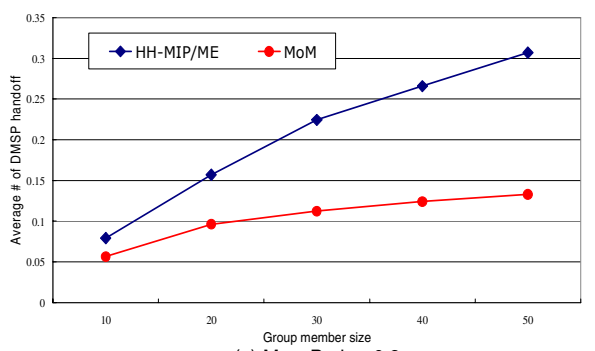

(a) MoveProb $=0.2$

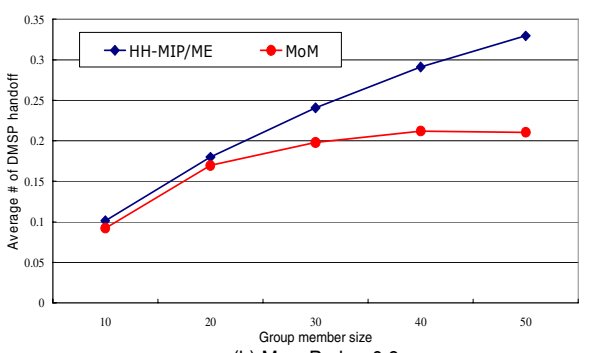

(b) MoveProb $=0.9$

Fig. 6. Number of DMSP handoff at different MoveProb

When we compare Fig. 6 (a) with (b), we find out a very interesting phenomenon that the value of average number of DMSP handoff in Fig. 6 (b) is higher than value in Fig. 6 (a). As we normalize the average number of DMSP handoff with number of MH handoff, the value must be the same at both figures. In Fig. 7, we run simulations to obtain total number of DMSP handoff in different mobility pattern with group size $=10$ for both protocols. In both simulations we find out that the value in y axis (total number of DMSP handoff) does not increase linearly in case of different mobility pattern. We assume that the phenomenon is caused by the complex mechanism in the DMSP selection.
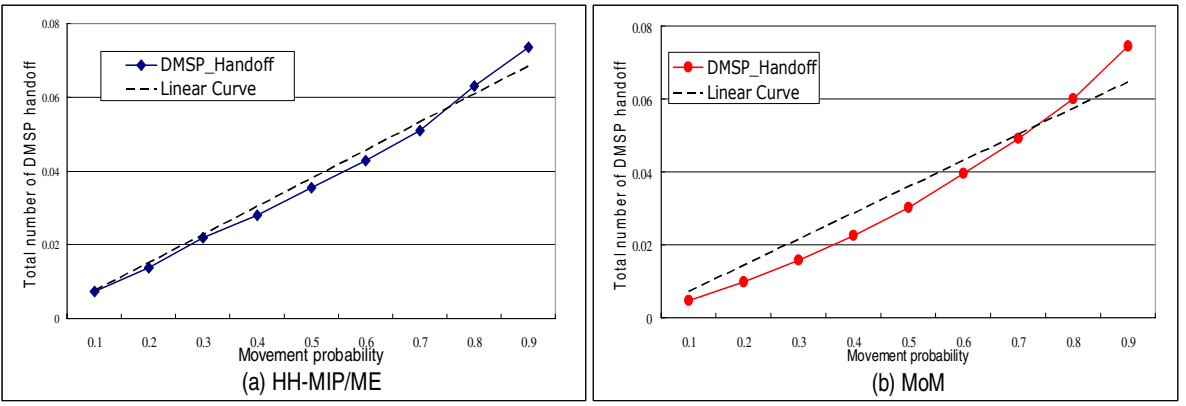

Fig. 7. HH-MIP/ME and MoM total number of DMSP Handoff

Fig. 8 shows the average DMSP handoff latency (in hop counts). The MoM has larger DMSP Handoff Latency because length of the path needs to update the new selected DMSP (HA) is longer in the MoM than HH-MIP/ME. The HH-MIP/ME has moderate DMSP Handoff Latency because MH's THA is resided near the MH current position. 


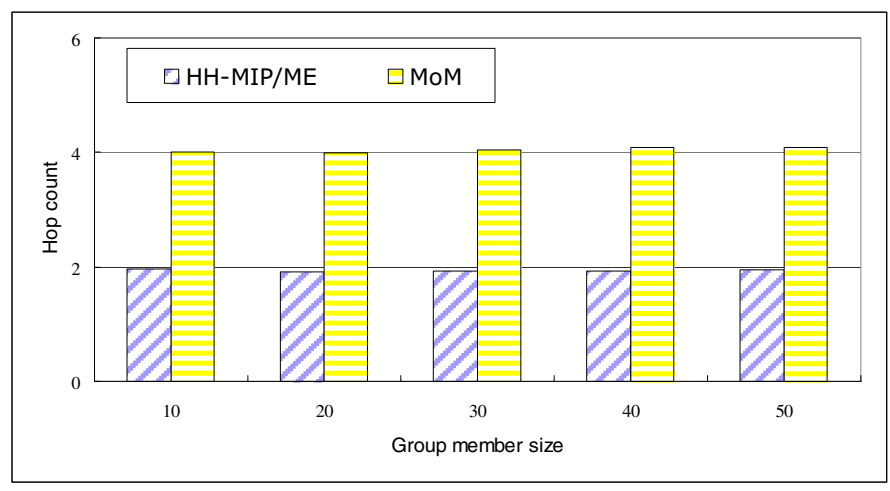

Fig. 8. DMSP handoff latency

\section{Conclusion}

The HH-MIP/ME as multicast extension of HH-MIP is presented in the paper. The $\mathrm{HH}-\mathrm{MIP} / \mathrm{ME}$ inherits the advantages of $\mathrm{HH}-\mathrm{MIP}$ because it is built on the top of $\mathrm{HH}$ MIP. The HH-MIP/ME uses its THA on behalf of MH to join the multicast group. Multicast packets are sent by multicast sender to MH's THA and the THA will tunnel to $\mathrm{MH}$ current position (FA). To avoid data duplication and tunnel convergence problem, the HH-MIP/ME uses DMSP concept. The simulation results also show that the HH-MIP/ME has better performance than existing protocols like the MoM and Remote Subscription.

Acknowledgments. This work was supported in part by the National Science Council, Taiwan, R.O.C., under grant NSC95-2219-E-260-004.

\section{References}

1. Akyildiz, I.F., Xie, J., Mohanty, S.: A Survey of Mobility Management in Next-Generation All-IP-based Wireless Networks. IEEE Wireless Communications, 16-28 (2004)

2. Perkins, C.E.: IP Mobility Support for IPv4. RFC 3344 (2002)

3. Johnson, D., Perkins, C.E., Arkko, J.: Mobility support in IPv6. RFC 3775 (2004)

4. Hwang, Y.-H., Chen, J.-Y., Yang, C.-C., Chen, W.-S.: A comparison between SIP and network layer mobility management protocols in wireless IP networks. In: Proceedings of Fifth IEE International Conference on 3G Mobile Communication Technologies, pp. 317321 (2004)

5. Campbell, A.T., Gomez, J., Kim, S., Turányi, Z.R., Valkó, A.G., Wan, C.Y.: Internet micromobility. Journal of High Speed Networks 11(3-4), 177-198 (2002)

6. Perkins, C.E., Johnson, D.B.: Route Optimization in Mobile IP. draft-ietf-mobileipoptim11.txt (2001)

7. Dell'Abate, M., De Marco, M., Trecordi, V.: Performance evaluation of Mobile IP protocols in a wireless environment. In: Proceedings of 1998 IEEE International Conference on Communications, pp. 1810-1816 (1998) 
8. Yu, L.-S., Yang, C.-C.: An Enhancement of Mobile IP by Home Agent Handover. In: Proceedings of IEEE 62nd Semiannual Vehicular Technology Conference (2005)

9. Chikarmane, V., et al.: Multicast support for mobile hosts using mobile IP: Design issues and proposed architecture. Mobile Networks and Applications 3(3), 365-379 (1998)

10. Harrison, T., et al.: Mobile Multicast (MoM) Protocol: Multicast Support for Mobile Hosts. ACM MOBICOM 97, 151-160 (1997)

11. Ye, M.-H., et al.: The Implementation of Multicast in Mobile IP. In: Proceedings of WCNC 2003, pp. 1796-1800 (2003)

12. Lin, C.-R.: Mobile Multicast Support in IP Networks. In: IEEE Global Telecommunication Conference, vol. 2, pp. 1935-1939 (2002)

13. Deering, S., et al.: An Architecture for Wide-Area Multicast Routing. In: Proceedings of ACM SIGCOMM Conference, pp. 126-135 (1994)

14. Fenner, W.: Internet Group Management Protocol Version 2. RFC 2236 (1997)

15. Deering, S.: Host Extensions for IP Multicasting. RFC 1112 (1989)

16. Yang, C.-C., Lin, K.-Y.: Distributed Mobile Tracking: A Novel Location Management Scheme for Routing Improvement in Cellular IP Networks. Journal of Computer Networks 43(2), 141-167 (2003) 